ARTICLE

\title{
Covalent assembly of nanoparticles as a peptidase-degradable platform for molecular MRI
}

\author{
Francisco Perez-Balderas ${ }^{1,2}$, Sander I. van Kasteren ${ }^{2, \dagger}$, Alaa A.A. Aljabali ${ }^{2,3, \dagger}$, Kim Wals ${ }^{2,4}$, Sébastien Serres ${ }^{1, \dagger}$, \\ Andrew Jefferson ${ }^{3}$, Manuel Sarmiento Soto ${ }^{1}$, Alexandre A. Khrapitchev', James R. Larkin ${ }^{1}$, Claire Bristow ${ }^{1}$, \\ Seung Seo Lee ${ }^{2, \dagger}$, Guillaume Bort ${ }^{2}$, Filippo De Simone ${ }^{2}$, Sandra J. Campbell ${ }^{1}$, Robin P. Choudhury ${ }^{3}$, \\ Daniel C. Anthony ${ }^{4, \star}$, Nicola R. Sibson ${ }^{1, \star} \&$ Benjamin G. Davis ${ }^{2, \star}$
}

Ligand-conjugated microparticles of iron oxide (MPIO) have the potential to provide high sensitivity contrast for molecular magnetic resonance imaging (MRI). However, the accumulation and persistence of non-biodegradable micron-sized particles in liver and spleen precludes their clinical use and limits the translational potential of MPIO-based contrast agents. Here we show that ligand-targeted MPIO derived from multiple iron oxide nanoparticles may be coupled covalently through peptide linkers that are designed to be cleaved by intracellular macrophage proteases. The synthesized particles possess potential characteristics for targeted MRI contrast agents, including high relaxivity, unappreciable sedimentation, clearance from circulation and no overt toxicity. Importantly, we demonstrate that these particles are rapidly degraded both in vitro and in vivo, and that the targeted probes can be used for detection of inflammation in vivo using MRI. This approach provides a platform for molecular MRI contrast agents that is potentially more suitable for translation to humans.

\footnotetext{
${ }^{1}$ Department of Oncology, Cancer Research UK \& Medical Research Council Oxford Institute for Radiation Oncology, University of Oxford, Oxford OX3 7DQ, UK. ${ }^{2}$ Department of Chemistry, Chemistry Research Laboratory, University of Oxford, Oxford OX1 3TA, UK. ${ }^{3}$ Department of Cardiovascular Medicine and Oxford Acute Vascular Imaging Centre, University of Oxford, John Radcliffe Hospital, Oxford OX3 9DU, UK. ${ }^{4}$ Department of Pharmacology, University of Oxford, Oxford OX13QT, UK. * These authors contributed equally to this work. † Present addresses: Gorlaeus Laboratory, Leiden Institute of Chemistry, Leiden University, Einsteinweg 55, The Netherlands (S.I.v.K.); Pharmacy Department, Yarmouk University, Irbid, Jordan (A.A.A.A.); School of Life Sciences, University of Nottingham, Nottingham NG7 2UH, UK (S.S.); School of Chemistry, Faculty of Natural and Environmental Sciences, University of Southampton, Highfield, Southampton SO17 1BJ, UK (S.S.L.). Correspondence and requests for materials should be addressed to D.C.A. (email: daniel.anthony@pharm.ox.ac.uk) or to N.R.S. (email: nicola.sibson@oncology.ox.ac.uk) or to B.G.D. (email: ben.davis@chem.ox.ac.uk).
} 
T he high magnetization and low toxicity of superparamagnetic iron oxide particles has led to their widespread use in biomedical and biological applications ${ }^{1-3}$, including $\mathrm{MRI}^{4,5}$, cancer therapy ${ }^{6}$, cell labelling ${ }^{2}$, biomolecule sensing ${ }^{8,9}$ and multimodal imaging ${ }^{10}$. These particles can be synthesized across a range of diameters, of which the ultrasmall superparamagnetic particles of iron oxide ${ }^{5}$ (USPIO; 20-50 nm in diameter) have been clinically used the most. The long circulation half-life of USPIO precludes rapid molecular imaging of target-specific binding owing to high background levels ${ }^{11}$. In contrast, microparticles of iron oxide (MPIO) have a short half-life $(<5 \mathrm{~min}$ (ref. 12)) in the circulation and greater contrastto-noise per particle than USPIO owing to their higher iron content $\left(0.26 \mathrm{pg}\right.$ iron per particle versus $1.1 \times 10^{-6} \mathrm{pg}$ iron per particle). Moreover, the increased surface area of MPIO (ca. $2-12 \mu \mathrm{m}^{2}$ ) compared with USPIO (ca. $0.005-0.03 \mu \mathrm{m}^{2}$ ) enables greater ligand valency, which can substantially increase binding affinities through multivalent effects ${ }^{13,14}$. In this context, the strong correlation between target quantification in tissue and contrast volume on non-invasive MRI points strongly towards the advantages of MPIOs ${ }^{15}$. The potential of MPIO for imaging diagnostically useful endovascular cellular events, such as inflammation and activated platelet adhesion, has already been highlighted in a range of animal models of important human disease, including multiple sclerosis ${ }^{16,17}$, thrombosis $^{18}$, atherosclerosis $^{19}$, renal ${ }^{15}$ and cerebral ischaemia ${ }^{16,20}$, cerebral malaria $^{21}$ and brain metastases ${ }^{22}$. If such MPIO could be synthesized in a biodegradable form, they have the potential to provide superior capabilities for molecular imaging of disease in humans (see Supplementary Note 1).

Although regulatory approval must of course be considered on a case-by-case basis, we consider that translation to clinical use requires a number of essential attributes: biocompatibility, high iron content, controlled biodegradability, functionalizable surface with multivalent capacity, appropriate shape to promote multivalent binding to the planar endothelial surface, short blood half-life, minimal non-specific accumulation and low tendency to agglomerate. Here, we describe a microparticle system designed through covalent assembly of multiple iron oxide nanoparticles (NPs), which combines the advantages of the micron-size iron particle range with biodegradability via the endogenous clearance $\mathrm{e}^{23-25}$ and degradation systems of the body (Fig. 1, Supplementary Fig. 1). These microparticles are therefore deserving of further research for their potentially enhanced utility as in vivo imaging agents and potential clinical application as iron-based molecular MRI contrast agents.

\section{Results}

mMPIO construction via iron oxide NP conjugation. Biodegradable multimeric MPIO (mMPIO) were covalently assembled from multiple colloidal iron oxide NPs of diameter $65 \mathrm{~nm}$ bearing an amine-functionalized dextran coat (Fig. 2). These common precursor particles (Supplementary Figs 2 and 3 and Supplementary Tables 1 and 2) were then elaborated into two complementary NP subtypes that could be readily coupled together. In the first subtype, carboxylate groups were created from amino groups using succinic anhydride to form carboxy$\mathrm{NPs}^{26}$ (Supplementary Fig. 3). In the second, peptide sequences were added to create peptido-NPs (Supplementary Fig. 3 and Supplementary Table 3). These peptide sequences were carefully designed to provide not only a suitable linker between monomer particles but also a linker that would be sensitive to specific intracellular degradative enzymes. Such proteases have previously been shown to display strong potential in, for example, targeted toxin release systems $s^{27,28}$ and are noted in differential regulation in some disease states ${ }^{29}$. The cathepsin proteins are the primary degradative enzymes in macrophages, which are the main site of sequestration of iron oxide particles in the liver and spleen on clearance from the circulation ${ }^{30-32}$. The proteolytic activity of lysosomal cathepsin B (EC 3.4.22.1) and L (EC 3.4.22.15) was tested against possible consensus peptide sequences $^{33-35}$. The most efficiently cleaved (Supplementary Figs 4-8) yet plasma stable (Supplementary Figs 9 and 10) peptide was chosen and incorporated. Stability of this peptide under serum conditions was also tested (Supplementary Figs 11-13); assays revealed degradation following clotting of blood but stability upon treatment with EDTA (Supplementary Fig. 13), suggesting susceptibility to metal-dependent proteases induced in the clotting cascade (e.g., Factor IXa and XIa). To stringently test the specificity of these chosen sequences we created four homologues of the cathepsin L-specific peptide Fmoc-Ahx-Ahx-FVRGAGE (Supplementary Fig. 4). In these homologues, key residues were varied or scrambled, and D-amino acids were used (including a 'mirror-image' peptide). When examined in detail, through the kinetics of cleavage combined with MS analysis (see Supplementary Figs 14-17 and Supplementary Table 5), these revealed much lower cleavage activity towards the scrambled and particularly the D-amino acid peptide, as expected.

Using the carboxy-NP and peptido-NP monomers, larger mMPIO were synthesized. This synthesis was performed using robust amide-forming chemistry in MES buffer $\mathrm{pH} 6.0$ via $N$-hydroxysulfosuccinimide (sulfoNHS) ester ${ }^{36}$. The resulting panel of mMPIO was characterized and optimized for key parameters, including size, yield and reactivity (Fig. 3a and Supplementary Table 4). Combined electron microscopy, transmission electron microscopy (TEM), atomic force microscopy (AFM), zeta potential and dynamic light scattering (DLS) measurements confirmed construction and the anticipated morphology and characteristics of our designed larger, multimeric particles (Fig. 3b and Supplementary Fig. 18 and Supplementary Tables 4 and 6). Composition and dextran content were further confirmed by chemical and elemental analyses. Importantly, by varying the reaction ratio of the two monomer types it was possible to change both the particle size and the nature of the surface reactive functional groups of the resulting MMPIO (Fig. 3a and Supplementary Table 4), which in turn would ensure that a broad range of targeting ligands could be conjugated. This approach, therefore, allowed us to readily create mMPIO bearing either an excess of amine (mMPIO- $\left.\mathrm{NH}_{2}\right)$ or those bearing an excess of carboxylic acid (mMPIO-COOH).

mMPIOs show low sedimentation and high MRI relaxivities. We have previously shown that microparticles of $0.5-1 \mu \mathrm{m}$ diameter combine favourable characteristics of high contrast effect and rapid blood phase clearance rate that are well suited to in vivo targeting studies ${ }^{16}$, by delivering efficient particle binding to the site of interest and low background signal at the time of MRI. At the same time, the MPIO are still much smaller than erythrocytes and not prone to microvascular plugging. mMPIO within this size range were efficiently and reliably constructed through modulation of the starting ratios of the two monomers (Fig. 3a). These mMPIO exhibited physicochemical advantages over equivalent monomeric iron-dextran MPIO $(\sim 0.7 \mu \mathrm{m}$ diameter; see Supplementary Methods for synthesis). In particular, the mMPIO showed very little tendency to precipitate, with sedimentation rates markedly lower than correspondingly sized monomeric particles and commercially available polystyrene-coated particles $(<1,51$ and $86 \%$ of sedimentation at $24 \mathrm{~h}$, respectively; 
(i) Inflammatory disease present
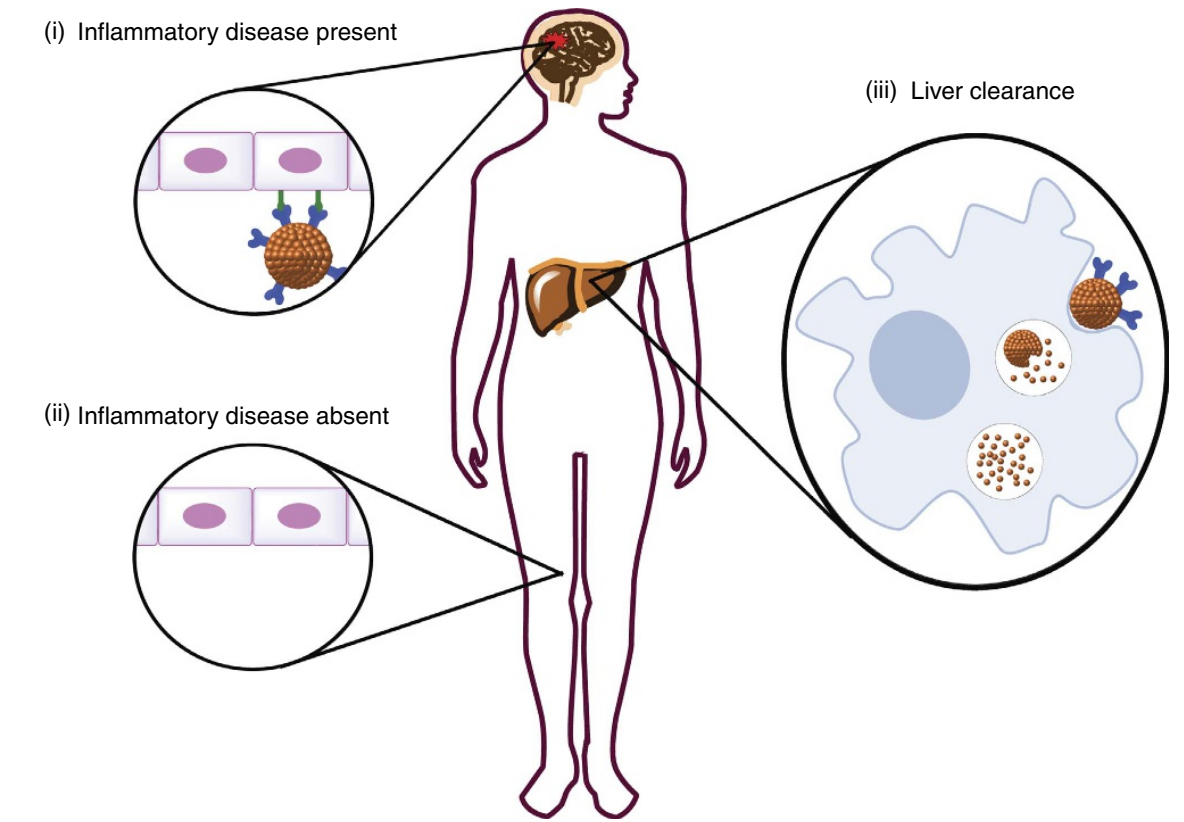

(iii) Liver clearance

Figure 1 | Molecular basis of mMPIO as a targeted MRI contrast agent. Intravenously injected targeted mMPIO bind to their target on the diseased endothelial surface (i), but do not bind to healthy endothelium (ii). The unbound mMPIO are rapidly cleared from blood. Thus, negligible background contrast effects are evident with mMPIO shortly after injection. mMPIO are efficiently taken up by macrophages (iii), and after internalization and fusion to lysosomes the internal peptide linkers are degraded. mMPIO are represented as brown sphere conglomerates, iron oxide nanoparticles are represented as brown spheres, targeting agents are represented in dark blue and endothelial surface disease markers are shown in green.

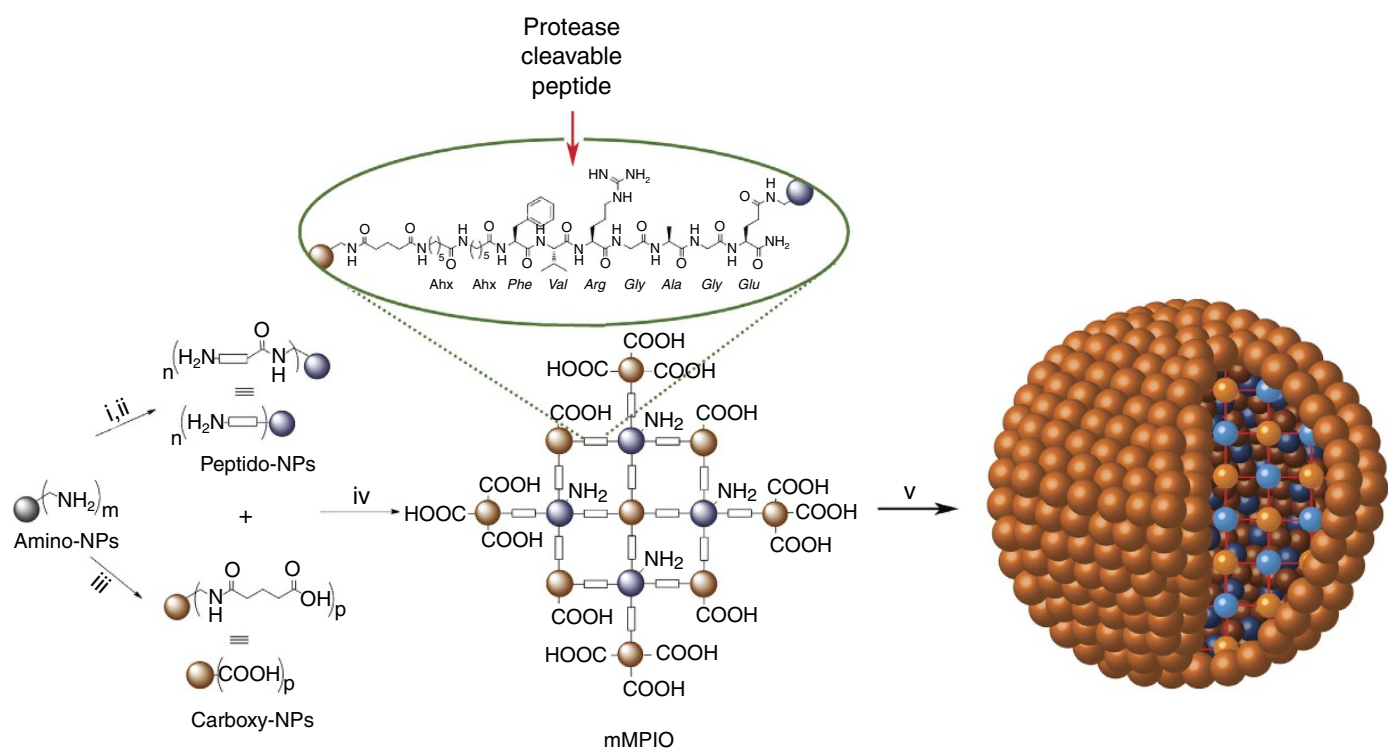

Figure 2 | Schematic representation of the synthesis of mMPIO. Grey spheres represent amine-functionalized dextran-coated iron oxide nanoparticles, blue spheres represent peptido-NPs and brown spheres represent carboxy-NPs. Conditions: (i) peptide 3, N-(3-dimethylaminopropyl)- $N^{\prime}$-ethylcarbodiimide hydrochloride (EDC) N-hydroxysuccinimide (NHS), 2-(N-morpholino)ethanesulfonic acid (MES) buffer (0.1 M pH6.0)/dimethylsulfoxide (DMSO) 1:1; (ii) piperidine/ $\mathrm{N}, \mathrm{N}$-dimethylformamide (DMF)/DMSO (2:3:5); (iii) succinic anhydride, sodium bicarbonate buffer (0.1 M pH 8.3)/DMSO 1:1;

(iv) EDC, sulfoNHS, MES buffer (0.1 M pH 6.0); (v) when an excess of carboxy-NPs are used an mMPIO with excess surface carboxylic acid functions is formed; when an excess of peptido-NPs is employed the MMPIO surface contains excess amine functions (not shown).

Supplementary Figs 19 and 20); such effects potentially increase the translational potential of the mMPIOs and may be due to crosslinking or surface charge changes (although they are consistent with many different surface potential levels; Supplementary Fig. 3). Moreover, the mMPIO displayed storage stability at $4{ }^{\circ} \mathrm{C}$ for more than 6 months (Fig. 3c). They also exhibited significantly higher $\mathrm{T}_{2}$ relaxivities $\left(194.5 \pm 4.5 \mathrm{mM}^{-1} \mathrm{~s}^{-1}\right.$ at $4.7 \mathrm{~T}$ and $196.5 \pm 4.9 \mathrm{mM}^{-1} \mathrm{~s}^{-1}$ at $7 \mathrm{~T}$; Fig. 3d and Supplementary Fig. 21 and Supplementary Table 7) than commercially available polystyrene-coated particles $\left(58.5 \pm 2.2\right.$ and $59.5 \pm 1.2 \mathrm{mM}^{-1} \mathrm{~s}^{-1}$ at 4.7 and $7 \mathrm{~T}$, respectively; Supplementary Table 7) that have previously ${ }^{16}$ demonstrated good contrast in vivo, potentially due to previously noted magnetic relaxation switch phenomena 9 . 
a

\begin{tabular}{ccc}
\hline & $\begin{array}{c}\text { Particle } \\
\text { ratio(a) }^{(\mathrm{a})}\end{array}$ & $\begin{array}{c}\text { Size } \\
(\mathrm{nm})^{(\mathrm{b})}\end{array}$ \\
\hline 1 & $1: 8$ & 250 \\
2 & $1: 7$ & 450 \\
3 & $1: 6$ & 711 \\
4 & $1: 5$ & 1,569 \\
5 & $1: 4.5$ & 2,279 \\
6 & $6: 1$ & 504 \\
7 & $5.5: 1$ & 808 \\
\hline
\end{tabular}

C

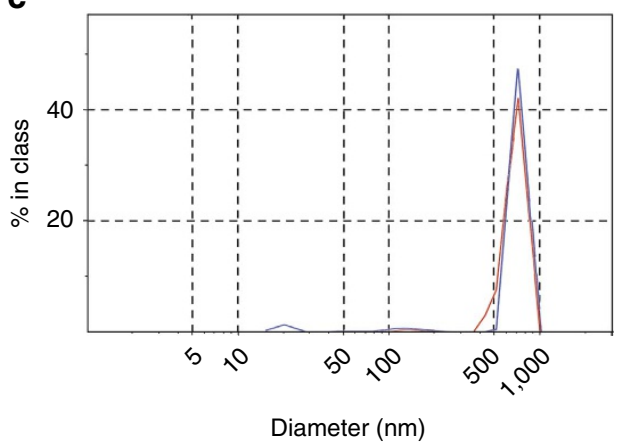

b

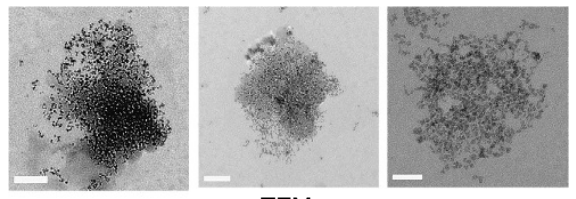

TEM

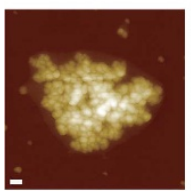

AFM

d

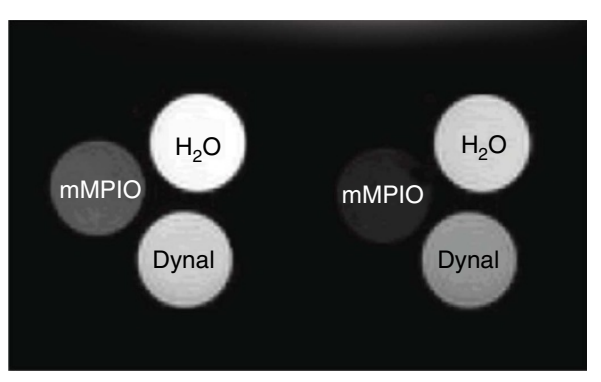

Figure 3 | Physicochemical characterization of mMPIO. (a) Size and yield of mMPIO as a function of carboxy-NP/peptido-NP ratio. ${ }^{[a]}$ Ratio between carboxy-NPs and peptido-NPs (w/w). ${ }^{[b]}$ Determined by dynamic light scattering (DLS). (b) Microscopic characterization of mMPIO by TEM (top three images showing different representative examples; scale bars left-to-right 100, 100 and $50 \mathrm{~nm}$ ), atomic force microscopy (bottom left, scale bar $50 \mathrm{~nm}$ ) and HRTEM (bottom middle and right; bottom right is the inset magnification from bottom middle; scale bars 50 and $5 \mathrm{~nm}$ ). (c) DLS size distribution graph of $\sim 700 \mathrm{~nm}$ mMPIO just after synthesis (red line) and after storage for 6 months (blue line). (d) $\mathrm{T}_{2}$-weighted images of the phantoms (iron concentration $=0.224 \mathrm{mM}$ ) at echo times $9.7 \mathrm{~ms}$ (left) and $16.3 \mathrm{~ms}$ (right).

mMPIO are biodegraded in vitro and in cellulo. Next, the biodegradability of the mMPIO was evaluated in vitro. Consistent with their molecular design, incubation of mMPIO with both cathepsins $\mathrm{B}$ and $\mathrm{L}$ efficiently degraded the peptide linker yielding the monomeric NPs from which they were built (Fig. 4a). Having demonstrated the biodegradable nature of the linker with respect to the appropriate enzymes, cellular uptake and intracellular degradation of the MMPIO were evaluated. Both amino-terminated mMPIO (mMPIO- $\left.\mathrm{NH}_{2}\right)$ and carboxylic acid-terminated mMPIO (mMPIO-COOH) (entries 3 and 7 from Fig. 3a, respectively) were labelled with the fluorophore AlexaFluor 488 cadaverine. These fluorescently labelled mMPIO were incubated with cultured macrophages. Confocal time-course experiments showed a consistent and clear reduction in the number of intracellular mMPIO over $12 \mathrm{~h}$ (Fig. 4b, Supplementary Figs 22 and 23 and Supplementary Movie 1). Further experiments (Supplementary Figs 24 and 25) showed that punctuate fluorescence in the intracellular compartment faded over time. After $72 \mathrm{~h}$, the fluorescence was homogeneously distributed and no large particles were detectable, suggesting complete degradation. By comparison, the equivalent large $(733 \mathrm{~nm})$ amino-terminated monomeric MPIO remained intact after $48 \mathrm{~h}$, with only slight degradation after $72 \mathrm{~h}$ (Supplementary Fig. 26), suggesting a considerably slower rate of degradation compared with the mMPIO. Cellular uptake of the mMPIO was confirmed and subcellular distribution of the degradation products was also analyzed in macrophage cell line (RAW 264.7) using TEM (see Supplementary Fig. 27) over $72 \mathrm{~h}$. Upon internalization into the distinctive cell morphology of the cells, mMPIO were clearly surrounded by visible membrane structures, suggesting anticipated formation of endolysosomal compartments within which the structure of the mMPIO degraded. This confirmed the uptake into phagosomes of high-intensity material containing iron and then its loss over time. In contrast, commercially available monomeric MPIO beads remained intact (with high iron content) throughout. We also constructed mMPIOs linked by peptides containing D-amino acids in the same sequence used for the degradable mMPIOs; consistent with the resistance shown by D-amino acid-containing peptides to cathepsins, these 'D'-mMPIO were not degraded (Supplementary Figs 28-32). These experiments further supported both the efficacy and the molecular mechanistic basis of the designed degradable 'L'-mMPIO system.

mMPIOs are not non-specifially retained and clear rapidly. Having confirmed in vitro degradation of the $\mathrm{mMPIO}$, the in vivo biodistribution and clearance of the MMPIO were evaluated histologically (Fig. 4c,d and Supplementary Figs 33 and 34). Both mMPIO- $\mathrm{NH}_{2}$ and the equivalent size, amino-terminated, monomeric MPIO were injected intravenously into naïve mice. Primary uptake of both the mMPIO and monomeric MPIO was evident in the liver $1 \mathrm{~h}$ after injection, and in both cases the particles were almost entirely cleared from this site by 7 days. Low-level mMPIO retention was also observed in the intestine and lung at $1 \mathrm{~h}$, although the level of particle staining was low compared with that observed in the liver (0.001 and 0.044 , respectively, versus $0.208 \%$ area stained) and, again, was negligible by 7 days. Similar low levels of monomeric MPIO retention were found in the intestine. In striking contrast, substantial monomeric MPIO retention was evident in the lungs, which was lower but still evident 14 days post-injection 
a

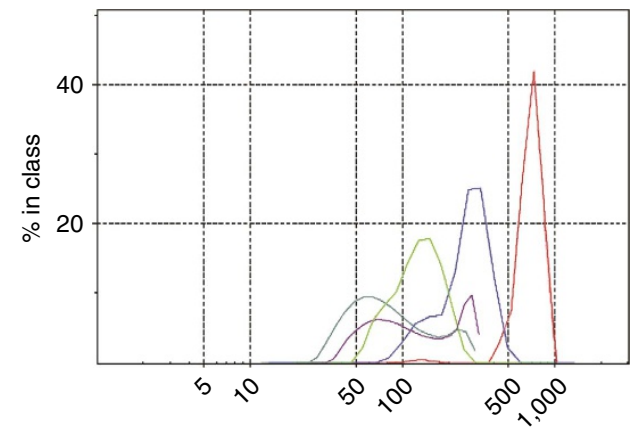

Diameter $(\mathrm{nm})$

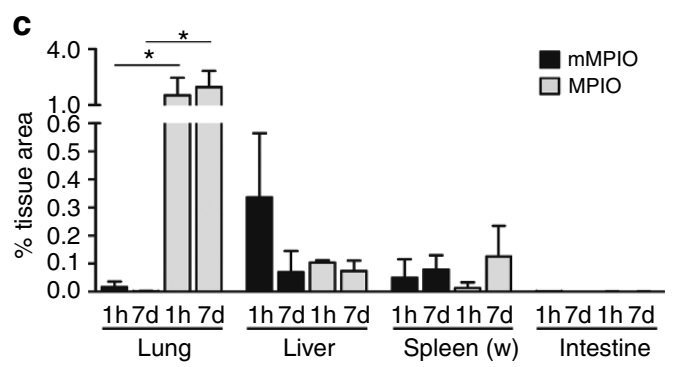

b
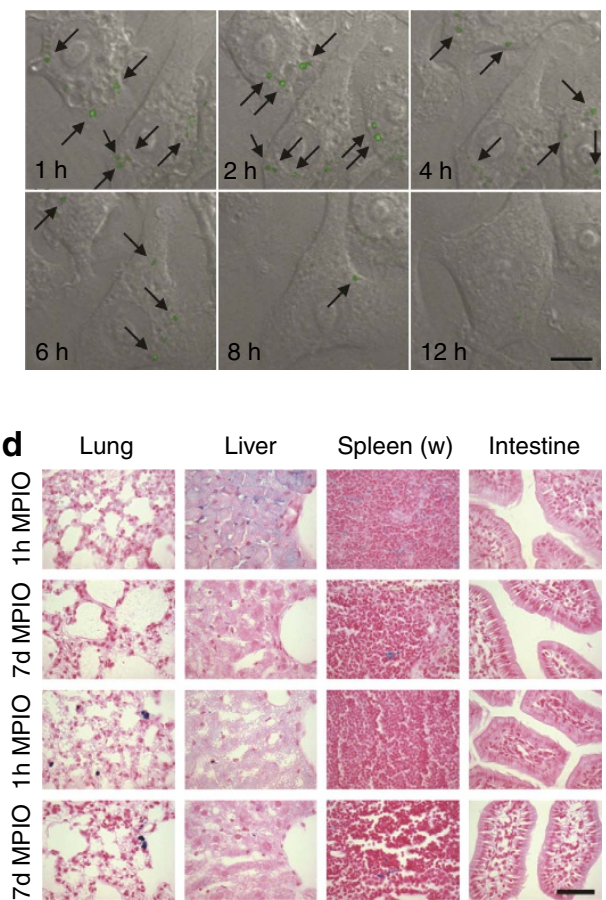

Figure 4 I In vitro and in vivo degradation studies. (a) DLS analysis of particle size distribution of mMPIO after addition of cathepsin L. Particle size was analysed at different time points $0 \mathrm{~h}$ (red line), $1 \mathrm{~h}$ (blue line), $4 \mathrm{~h}$ (light green), $8 \mathrm{~h}$ (purple) and $24 \mathrm{~h}$ (dark green). Experiments were performed in triplicate. (b) Time-course montage of particle degradation by the murine macrophage cell line RAW264.7 obtained by live cell confocal imaging. Visible particles are indicated by black arrows. Experiments were performed in triplicate. Scale bar $10 \mu \mathrm{m}$. (c) Graph to show distribution of ca. $700 \mathrm{~nm}$ mMPIO and MPIO in different organs $1 \mathrm{~h}$ and 7 days after intravenous injection ( $n=3$ per group). Data are mean \pm s.d. for three fields per organ. ${ }^{\star} P<0.05$ based on one-way ANOVA on a tissue-wise basis followed by Newman-Keuls post-hoc tests. (d) Images of immunohistochemical sections taken from different organs $1 \mathrm{~h}$ and 7 days after intravenous injection of either mMPIO or MPIO. Sections have been stained with Prussian Blue to identify iron and counterstained with Nuclear Fast Red. Magnification is $\times 400$, scale bar $=50 \mu \mathrm{m}$.

(ca. $1 \%$ tissue area). The lung is known to play an important role in removing blood-borne foreign bodies via adherent phagocytes and endothelial cells, and particles cleared in this way are passed through the endothelium to accumulate in macrophages in the alveolar interstitium ${ }^{37}$. Thus, it might be expected that MPIO would also be cleared from the circulation by this pathway. Unlike the MPIO, however, the mMPIO were not cleared to any appreciable extent via the lungs (cf. $1 \mathrm{~h}$ data; Fig. 4c,d and Supplementary Fig. 34), reflecting their different physicochemical properties. Very low and constant levels of iron staining were evident in the white pulp of the spleen in both mMPIO- and MPIO-injected animals (0.1-0.2\% tissue area), while the very high intrinsic iron levels in the red pulp of the spleen precluded quantitation of the small increases arising from mMPIO/MPIO accumulation. No appreciable retention of the mMPIO or monomeric MPIO was found in heart, brain or kidney $(<0.0005 \%$ of tissue area; Supplementary Fig. 33). Importantly, for our goal of creating a flexible particle system, chemical surface functionality did not alter in vivo distribution; no appreciable differences were seen in animals injected with mMPIO-COOH compared with those injected with mMPIO- $\mathrm{NH}_{2}$.

To assess whether labelling of the mMPIO with targeting antibodies altered the clearance profile and to obtain formal toxicological data, preclincial studies were conducted (Sequani; see Supplementary Methods) in which mice were injected with mMPIO conjugated to a humanized antihuman-vascular cell adhesion molecule (VCAM)-1 antibody ( $\alpha$ huVCAM) with cross-reactivity to mouse ( $\alpha$ huVCAM-
mMPIO). All values for clinical observations (body weight, organ weights, macroscopic histology) were within normal ranges, and no blood chemistry (Supplementary Tables 9 and 10), haematological (Supplementary Tables 11 and 12) or histological findings (Supplementary Fig. 35) were of toxicological significance. In accord with our findings above, a very low level of diffuse iron staining was found in the liver 2 days after administration of ahuVCAM-mMPIO in 5/6 mice, which was no longer evident by 14 days post- $\alpha$ huVCAM-mMPIO injection (Supplementary Fig. 35). In-house quantitative analyses confirmed the absence of particulate iron deposits (i.e., undegraded mMPIO) at both 2 and 14 days post- $\alpha$ huVCAM-mMPIO injection, indicating degradation within the first $48 \mathrm{~h}$ period. No $\alpha$ huVCAM-mMPIO retention was evident in any other tissue, or in control animals, at either time point. Thus, the biodistribution profile of the mMPIO remained the same when conjugated to a targeting antibody. Since VCAM-1 is not highly expressed on vascular endothelium under normal conditions, accumulation in tissue beds expressing VCAM-1 other than the brain was not examined, as this would have required different disease models. No evidence of infarction or inflammation was found in any of the organs studied in any of the above studies, up to 14 days post-mMPIO injection.

Antibody-targeted mMPIOs allow molecular imaging. Having established biodistribution profiles, the utility of this platform to create targeted mMPIO as tools for molecular imaging was tested. The diversity of functional groups in the mMPIO, which is a consequence of their multimeric assembly, also allowed 

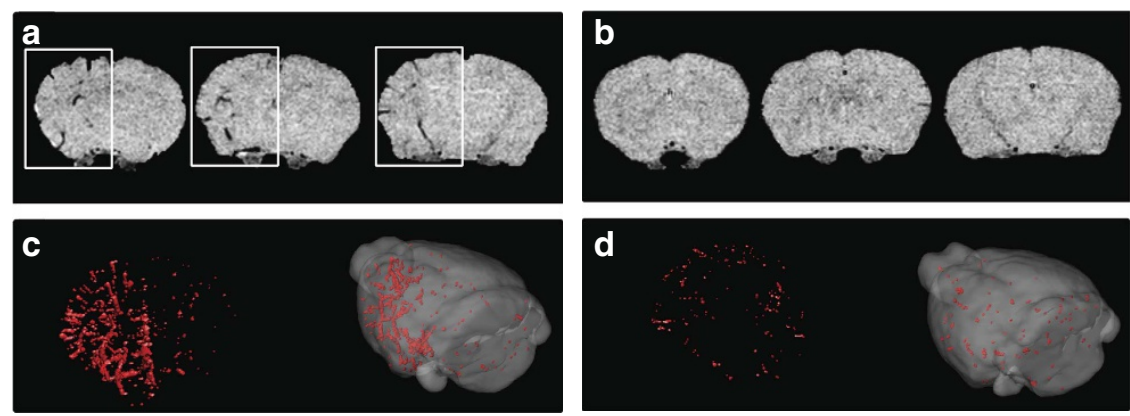

e

f

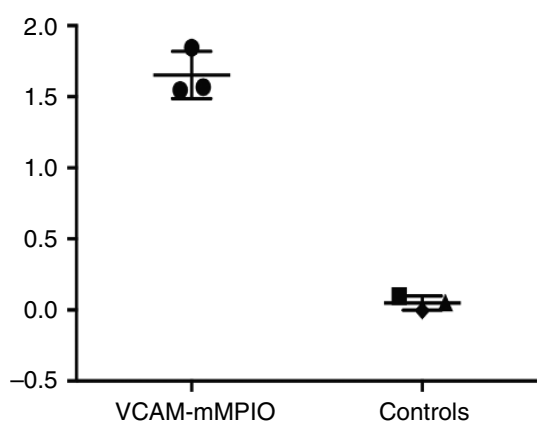

(i)
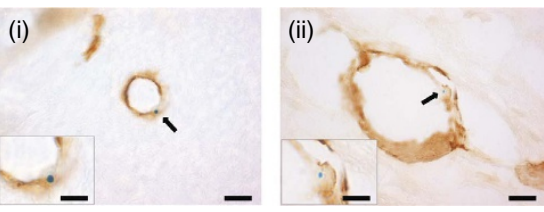

g
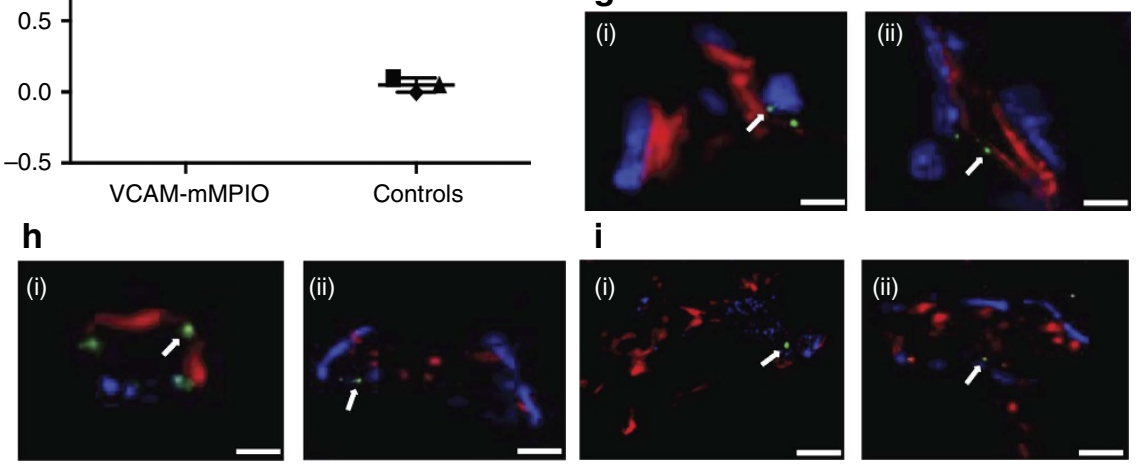

i
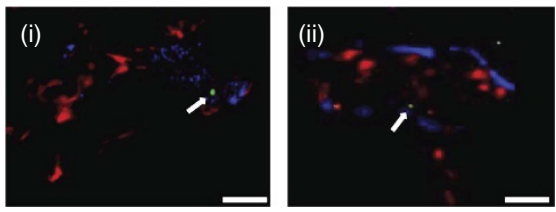

Figure 5 | In vivo VCAM-1-targeting experiments. (a,b) Selected $\mathrm{T}_{2}{ }^{*}$-weighted images from 3D data sets obtained from mice injected intrastriatally with IL-1 $33 \mathrm{~h}$ before intravenous injection of either (a) $\alpha$ VCAM-AF488-mMPIO or (b) control non-targeted IgG-AF488-mMPIO. Focal hypointense areas (black) in the left hemisphere reflect the specific $\alpha$ VCAM-AF488-mMPIO retention on acutely activated vascular endothelium (a), with negligible contrast effects evident in either the contralateral control hemisphere or in the animal injected with IgG-AF488-mMPIO (b). Box indicates the injected hemisphere. (c,d) $3 \mathrm{D}$ reconstructions of the mMPIO-induced hypointensities (coloured in red) from mice injected with either $\alpha$ VCAM-AF488-mMPIO

(c) or IgG-AF488-mMPIO (d) shown both as a stacked plot (left) and within the mouse brain frame of reference (right). Negligible contrast effects are evident in the animal injected with IgG-AF488-mMPIO. (e) Graph to show volumes of hypointensities in $\alpha$ VCAM-AF488-mMPIO-injected mice (black circles; $n=3$ ) and controls (black square, IL-1 + IgG-AF488-mMPIO; black triangle, saline + IgG-AF488-mMPIO; black diamond,

naive $+\alpha$ VCAM-AF488-mMPIO). Data are shown as specific contrast (left-right difference) for each animal; note negligible specific contrast is apparent in the control mice. Each data point represents an individual mouse; since this was a proof-of-principle experiment to demonstrate in vivo contrast effects, statistical analysis was not performed. (f-i) Immunohistochemical and immunofluoresence images of brain sections taken from IL-1 $\beta$-injected mice. f $(\mathrm{i}$, ii) Immunohistochemical sections showing VCAM-1 expression (brown) co-localized with aVCAM-AF488-mMPIO (Prussian Blue staining). Epifluorescence ( $\mathbf{g}(\mathrm{i})$ ) and confocal ( $\mathbf{g}$ (ii)) images further demonstrate adherence of $\alpha$ VCAM-AF488-mMPIO (green) to VCAM-1-positive (red) vessels. Nuclei are stained with DAPI (blue). Additional alkaline phosphatase staining enabled co-localization of $\alpha$ VCAM-AF488-mMPIO (green) with VCAM-1 (blue) and vessels (laminin-red) to be confirmed using both epifluorescence (h (i)) and confocal (h (ii) and $\mathbf{i}(\mathrm{i}, \mathrm{ii})$ ) microscopy. Arrows indicate $\alpha$ VCAM-AF488-mMPIO. Scale bars $=10 \mu \mathrm{m}(\mathbf{f}(\mathrm{i}, \mathrm{ii}) ; \mathbf{h}(\mathrm{ii}) ; \mathbf{i}(\mathrm{i}, \mathrm{ii}))$ and $5 \mu \mathrm{m}(\mathbf{g}(\mathrm{i}, \mathrm{ii}) ; \mathbf{h}(\mathrm{i}))$.

ready orthogonal labelling such that different moieties could be incorporated via different functional groups (Supplementary Fig. 36). In this way, mMPIO were created that contained both multiple fluorescent labels (AF488) in the inner core of the particle and high levels of surface, targeting moieties. Particles were synthesized bearing either an anti-VCAM-1 antibody $(\alpha$ VCAM) or a corresponding IgG control antibody $(\alpha$ VCAMAF488-mMPIO and IgG-AF488-mMPIO, respectively). Both targeted particles exhibited similarly high surface antibody density (Supplementary Fig. 37). As expected, aVCAM-AF488mMPIO, but not IgG-AF488-mMPIO, showed high binding capacity towards activated endothelial cells in vitro (Supplementary Fig. 38).

Finally, the potential of the $\alpha$ VCAM-AF488-mMPIO for in vivo molecular MRI was evaluated in a mouse model of cerebral inflammation. Mice were injected intracerebrally with interleukin-1 $\beta$ (IL-1 $\beta$ ) in the left striatum to induce endothelial activation and VCAM-1 expression ${ }^{16}$. In animals subsequently injected intravenously with aVCAM-AF488mMPIO, a marked contrast effect was evident in the $\mathrm{T}_{2}{ }^{*}$-weighted images, manifest as focal hypointensities in the IL-1 $\beta$-injected hemisphere (Fig. 5a,c, Supplementary Fig. 39a and Supplementary Movie 2). Notably, the contrast effect was unilateral with no non-specific mMPIO-induced hypointensities in the non-injected hemisphere. Negligible contrast effects arising from mMPIO retention were present in any of the controls: (i) naïve mouse injected intravenously with $\alpha$ VCAM-AF488-mMPIO; (ii) mouse injected intracerebrally with saline and intravenously with $\alpha$ VCAM-AF488-mMPIO; and (iii) mouse injected intracerebrally with IL-1 $\beta$ and 
intravenously with the non-targeted IgG-AF488-mMPIO (Fig. 5b,d, Supplementary Fig. 39b and Supplementary Movie 3). Quantitative analyses of the volumes of hypointensity induced by $\alpha$ VCAM-AF488-mMPIO binding yielded substantially greater volumes in the IL- $1 \beta$-injected animals than in any of the control animals (Fig. 5e). Subsequent $\mathrm{T}_{1}$-weighted images acquired after intravenous administration of the passive contrast agent gadolinium-DTPA revealed no areas of contrast enhancement in any animal, verifying that the blood-brain barrier was intact. Taken together, these data indicate specific binding of $\alpha$ VCAM-AF488-mMPIO to acutely activated endothelium in the absence of blood-brain barrier breakdown.

Following the in vivo MRI experiments, co-localization of VCAM-1 expression and $\alpha$ VCAM-AF488-mMPIO binding was verified both immunohistochemically and by immunofluorescence (Fig. 5f-i). Immunohistochemical analysis demonstrated upregulation of VCAM-1 in the IL- $1 \beta$-injected, but not the contralateral, hemisphere. Subsequent Prussian Blue detection of iron revealed the presence of bound $\alpha$ VCAM-AF488-mMPIO in VCAM-1-positive vessels (Fig. 5f). Both epifluorescence and confocal microscopy of the brain sections further confirmed successful co-localization of the $\alpha$ VCAM-AF488-mMPIO with both VCAM-1 and laminin, indicating association of the targeted mMPIO with VCAM-1-positive vessels (Fig. 5g-i).

\section{Discussion}

We have demonstrated here some potential advantages conferred by the use of covalent linkages for the synthesis of mMPIO that include control of size and enhanced stability. These are both prerequisites for clinical use of the agent that are not provided by, for example, current methodologies based on non-covalent linkages 8,9 .

We have demonstrated here these mMPIOs as intravascular agents. In the system we propose here, molecular targeting is determined by a surface-displayed binding agent on the particle and an appropriate 'biomarker' binding partner on the cell surface. We should add the clear caveat that we have only tested a single targeting antibody type (anti-VCAM); other important target sites also exist (e.g., interstitial space or poorly vascularized tumour cells) for imaging on which they might also be tested in the future. However, at such sites targeted agents may lose their molecular selectivity since they can also accumulate passively.

The choice of a cathepsin-cleavable peptide as the linker ensured rapid degradation of the mMPIO once sequestered by macrophages, primarily within the liver following clearance from circulation $^{30,31}$, while particles that associate with their target remain unaltered and functional. Rapid sedimentation, slow degradation and mechanical retention in organs ${ }^{23,24}$, such as the lung, make the corresponding monomeric MPIO particles unsuitable for use in man. In contrast, the mMPIO appear to possess properties that are useful for clinical application of molecular imaging, such as high relaxivity (see Supplementary Table 8), unappreciable sedimentation rate, rapid degradation, no overt toxicity and fast clearance from circulation. Owing to these mechanistic differences, unlike toxic particles where dose would be rapidly limiting, excesses of mMPIO can advantageously be used. In this way, percentage binding can be even tuned accordingly, since any excess that does not bind is non-toxic, cleared rapidly and degraded. This therefore provides both a vital mechanistic and potential translational advantage. Thus, while key additional translational hurdles will need to be considered in even greater detail for future development and regulatory approval (i.e., stability, reproducibility, dispersity), we believe that these proof-of-principle experiments with mMPIO might provide a promising platform for the clinical use of molecular MRI contrast agents.

\section{Methods}

General considerations. All animal experiments were authorized by the UK Home Office. Chemical abbreviations and details of the equipment employed are given in Supplementary Methods.

Synthesis of dextran covered NPs. About $5 \mathrm{mmol}$ of $\mathrm{FeCl}_{3} \cdot 6 \mathrm{H}_{2} \mathrm{O}$ and 9,10 or $12 \mathrm{~g}$ of dextran average mol. wt. 9,000-11,000 (SigmaAldrich, cat. Nr. D9260, lot Nr. 1331472 or 1382459) or $7.3 \mathrm{~g}$ of Dextran 10 Pharmaceutical Quality (Pharmacosmos, batch Nr. HX4271) were dissolved in $20 \mathrm{ml}$ of water and deoxygenated thoroughly by repeated cycles of vacuum assisted by sonication and argon flushing. After the first deoxygenation cycle, $3 \mathrm{mmol}$ of $\mathrm{FeCl}_{2} \cdot 4 \mathrm{H}_{2} \mathrm{O}$ in $5 \mathrm{ml}$ of water was added and the solution was deoxygenated by the above procedure (four times). While being stirred with an overhead stirrer at 600 r.p.m., $\mathrm{NH}_{4} \mathrm{OH}$ $(4 \mathrm{ml}, 25 \%)$ was added at a rate of $168 \mathrm{ml} \mathrm{h}^{-1}$. The reaction was heated to $80^{\circ} \mathrm{C}$ and then stirred at this temperature for $1 \mathrm{~h}$. The solution was cooled and placed in a SpectraPor membrane (MWCO 100,000) leaving some space for volume increase. The solution was dialysed against $5 \mathrm{l}$ of water for $21 \mathrm{~h}$ with water changes at times 1,2 and $4 \mathrm{~h}$. Iron content was measured spectrophotometrically at $410 \mathrm{~nm}$ after acid dissolution (6 $\mathrm{M} \mathrm{HCl}(\mathrm{aq})$ ) and oxidation (3\% hydrogen peroxide solution) for $1 \mathrm{~h}$ (ref. 38), and adjusted to a final concentration of $10 \mathrm{mg}$ Fe per ml using a Vivaspin centrifugal unit (MWCO 30.000). An aliquot $(10 \mu \mathrm{l})$ was diluted with $700 \mu \mathrm{l}$ of phosphate-buffered saline (PBS) and particle size was determined by DLS. A sample ( $5 \mathrm{mg}$ of iron) was freeze dried and elemental analysis was determined (Supplementary Methods and Supplementary Table 1).

Synthesis of amino-terminated NPs. Twenty millilitres of dextran covered NPs $\left(10 \mathrm{mg} \mathrm{ml}^{-1}\right)$ were placed into a $250-\mathrm{ml}$ round flask equipped with a $30 \times 16 \mathrm{~mm}$ oval stirrer bar. While the solution was stirred at 500 r.p.m., $36.7 \mathrm{ml}$ of $\mathrm{NaOH} 5 \mathrm{M}$ was added at a rate of $168 \mathrm{ml} \mathrm{h}^{-1}$. After that, $13.3 \mathrm{ml}$ of epichlorohydrin $(20 \mathrm{ml}$ in case of Phamacosmos HX4271 dextran, results 'k-l' Supplementary Table 1) was added at a rate of $94 \mathrm{ml} \mathrm{h}^{-1}$. The mixture was stirred at 1,000 r.p.m. for $7 \mathrm{~h}$ and then $20 \mathrm{ml}$ of $\mathrm{NH}_{4} \mathrm{OH}(25 \%)$ was added at a rate of $168 \mathrm{ml} \mathrm{h}^{-1}$. The mixture was stirred at 1,000 r.p.m. for $14 \mathrm{~h}$ and then was placed in a SpectraPor 2 or $100 \mathrm{kDa}$ cutoff membrane leaving some space for volume increase. The solution was then dialysed against 51 of water for $21 \mathrm{~h}$ with water changes at times 1,2 and $4 \mathrm{~h}$ and the dialysate concentrated on a Vivaspin 15 unit (MCWO 30.000) to $15 \mathrm{mg}$ Fe per ml. A sample (5 mg of iron) was freeze dried and elemental analysis was determined (Supplementary Methods and Supplementary Table 2).

Synthesis of carboxylic acid terminated NPs. Succinic anhydride in dimethyl sulfoxide (DMSO) $\left(4 \mathrm{ml}, 15 \mathrm{mg} \mathrm{ml}^{-1}\right)$ was added to a solution of amino-NPs $(8 \mathrm{ml}, 10 \mathrm{mg}$ Fe per $\mathrm{ml})$ in sodium bicarbonate buffer $100 \mathrm{mM}$ pH 8.3 . Note: The reaction is exothermic and a nitrogen-filled balloon with a syringe was placed on the tube. The mixture was shaken for $3 \mathrm{~h}$, then $4 \mathrm{ml}$ of succinic anhydride in DMSO $\left(15 \mathrm{mg} \mathrm{ml}^{-1}\right)$ was added and the mixture shaken for a further $3 \mathrm{~h}$. The sample was dialyzed in a $10,000 \mathrm{Da}$ dialysis membrane against $5 \mathrm{l}$ of water for $21 \mathrm{~h}$ with water changes at times 1,2 and $4 \mathrm{~h}$ and was concentrated in a Vivaspin 20 unit (MCWO 30.000) to $15 \mathrm{mg}$ Fe per ml.

Peptide synthesis. SPPS peptide synthesis was performed under standard Fmoc-conditions using HBTU/HOBt as an activator and DIPEA as a base catalyst. Deprotection and couplings were performed under microwave irradiation employing a single coupling protocol except for the first residue, which was introduced by double coupling. Peptide cleavage was performed using TFA/TIS/ $\mathrm{H}_{2} \mathrm{O}$ 95:2.5:2.5 (10 ml per g resin). The solvent was partially evaporated and the peptide was precipitated by addition of a cold mixture of diethylether/ hexane 1:1, centrifuged and washed again with the same mixture. The peptide was homogenized, dried under high vacuum for $3 \mathrm{~h}$, re-dissolved in water/acetonitrile/ DMSO/TFA 70:15:5:0.1 and purified by preparative RP chromatography. This product was dissolved in $2 \%$ DMSO in water and analysed by high-performance liquid chromatography (HPLC). For structure of peptides, see Supplementary Fig. 4. Peptides were characterized by HPLC, high-resolution mass spectrometry and tandem mass spectrometry (MS/MS) (Supplementary Figs 40-47 and Supplementary Tables 13-17). The proteolytic activity of the cathepsins on the peptides was analyzed by HPLC (Supplementary Methods).

Synthesis of peptide covered NPs (peptido-NPs). A solution of EDC in water $\left(0.84 \mathrm{ml}, 38 \mathrm{mg} \mathrm{ml}^{-1}\right)$ was added to a solution of peptide $3(200 \mathrm{mg})$ and NHS $(24 \mathrm{mg})$ in DMSO $(3.7 \mathrm{ml})$. This solution was incubated for $5 \mathrm{~min}$ at r.t. and then added to a solution of $20 \mathrm{mg}$ of amino-NPs in $3.3 \mathrm{ml}$ of MES buffer $0.1 \mathrm{M}$ $\mathrm{pH}$ 6.0. The solution was shaken for $3 \mathrm{~h}$ at room temperature (r.t.), and then split into two $50 \mathrm{ml}$ centrifuge tubes. Particles were precipitated by addition of $45 \mathrm{ml}$ of acetonitrile, centrifuged for $20 \mathrm{~min}$ at $3250 \mathrm{~g}$ and the supernatant was carefully 
discarded taking care not to disturb the precipitate. The precipitate was re-suspended in $5 \mathrm{ml}$ of DMSO, an aliquot $(100 \mu \mathrm{l})$ was taken and Fmoc analysis was performed (Supplementary Methods). The rest of the sample was precipitated again by addition of $45 \mathrm{ml}$ of acetonitrile and centrifuged as before. Samples were re-suspended in $5 \mathrm{ml}$ of DMSO and $5 \mathrm{ml}$ of $40 \%$ piperidine in DMF was added. The samples were shaken for $30 \mathrm{~min}$ after which the particles were precipitated by addition of $45 \mathrm{ml}$ of 1,4-dioxane. Samples were re-suspended in $5 \mathrm{ml}$ of DMSO and the deprotection step was repeated. Precipitate was re-suspended in water, dialyzed in a 10.000 MWCO dialysis membrane against $5 \mathrm{l}$ of water for $21 \mathrm{~h}$ with water changes at times 1,2 and $4 \mathrm{~h}$ and was concentrated in a Vivaspin 6 unit (MCWO 30.000) to $15 \mathrm{mg}$ Fe per ml (Supplementary Table 3). An essentially similar method was used for the synthesis of D-peptido-NPs containing D-amino acids in the peptide.

\section{Synthesis of amino-terminated mMPIOs ( $\left.\mathbf{m M P I O}-\mathrm{NH}_{2}\right)$. A solution of} carboxy-NPs $(25.5 \mu \mathrm{l}, 11.2 \mathrm{mg}$ Fe per ml, $0.285 \mathrm{mg} \mathrm{Fe})$ in MES buffer $0.1 \mathrm{M}$ $\mathrm{pH} 6.0$ was activated by sequential addition of sulfoNHS ( $2 \mu \mathrm{l}, 1.5$ eq. respect to the $\mathrm{N}$ content of its amino-NPs precursor; see Supplementary Table 2) and of $\operatorname{EDC}(1 \mu \mathrm{l}, 1.2$ eq. respect to the $\mathrm{N}$ content of its amino-NPs precursor; see Supplementary Table 2) in MES buffer $0.1 \mathrm{M} \mathrm{pH}$ 6.0. The resulting solution was shaken and incubated at r.t. for $5 \mathrm{~min}$ and added to a solution of peptido-NPs $\left(171 \mu \mathrm{l}, 10 \mathrm{mg} \mathrm{ml}^{-1}, 1.71 \mathrm{mg} \mathrm{Fe}\right)$ in MES buffer $0.1 \mathrm{M} \mathrm{pH} 6.0$. The reaction was shaken at $55^{\circ} \mathrm{C}$ for $24 \mathrm{~h}$ at 1,400 r.p.m.. Once the reaction had finished the sample was diluted and purified by dialysis and either by magnetic pelleting (Supplementary Methods) or by sucrose gradient (Supplementary Methods). An aliquot $(2 \mu \mathrm{l})$ was redispersed in $700 \mu \mathrm{l}$ of PBS and size was measured by DLS (Supplementary Table 4). An essentially similar method was used for the synthesis of the D-amino-acid-mMPIOs from D-peptido-NPs.

Synthesis of carboxy-terminated mMPIOs (mMPIO-COOH). A solution of carboxy-NPs $(122 \mu \mathrm{l}, 15.3 \mathrm{mg} \mathrm{Fe}$ per ml, $1.71 \mathrm{mg} \mathrm{Fe})$ in MES buffer $0.1 \mathrm{M}$ $\mathrm{pH} 6.0$ was activated by sequential addition of sulfoNHS $(15 \mu \mathrm{l}, 1.5$ eq. respect to the N content of its amino-NPs precursor; see Supplementary Table 2) and of EDC ( $10 \mu \mathrm{l}, 1.2$ eq. respect to the $\mathrm{N}$ content of its amino-NPs precursor; Supplementary Table 2) in MES buffer $0.1 \mathrm{M} \mathrm{pH} \mathrm{6.0.} \mathrm{The} \mathrm{resulting} \mathrm{solution} \mathrm{was}$ shaken and incubated at r.t. for $5 \mathrm{~min}$ and added to a solution of peptido-NPs (22.8 $\mu \mathrm{l}, 12.5 \mathrm{mg}$ per $\mathrm{ml}, 0.285 \mathrm{mg} \mathrm{Fe}$ ) in MES buffer $0.1 \mathrm{M} \mathrm{pH}$ 6.0. The reaction was shaken at $55^{\circ} \mathrm{C}$ for $24 \mathrm{~h}$ at 1,400 r.p.m.. Once the reaction had finished the sample was diluted and purified by dialysis and either by magnetic pelleting (Supplementary Methods) or by sucrose gradient (Supplementary Methods). An aliquot $(2 \mu \mathrm{l})$ was redispersed in $700 \mu \mathrm{l}$ of PBS and particle size measured (Supplementary Table 4).

Sedimentation of particles. A solution of mMPIO or $733 \mathrm{~nm}$ MPIO (for synthesis see Supplementary Methods) ( $0.11 \mathrm{mg}$ Fe per ml) was placed, after vortexing and sonication, in a quartz cuvette equipped with PTFE stopper. Absorption was measured at $\lambda=500 \mathrm{~nm}$ for $24 \mathrm{~h}$ at $5 \mathrm{~min}$ time intervals.

Relaxivity of compounds. Phantoms were prepared by addition of $0.75 \mathrm{ml}$ of an agarose solution (12\%) to $0.75 \mathrm{ml}$ of serial dilutions of the contrast agent in a Nalgene Cryoware vial. Samples were centrifuged at $1,000 \mathrm{~g}$ for $5 \mathrm{~min}$ to eliminate air bubbles. MRI experiments were performed on Magnex 4.7 or 7.0 T superconductive magnet driven by Varian DirectDrive spectrometer (Magnex Scientific and Varian Inc.; subsidiaries of Agilent Technologies, Santa Clara, CA, USA). A spin-echo sequence was used to acquire $\mathrm{T}_{2}$ and $\mathrm{T}_{1}$ maps. Single slice images were acquired with a matrix size of $128 \times 128$ pixels in all cases, corresponding to voxel dimensions of $0.4 \times 0.4 \times 5.0 \mathrm{~mm}$. $\mathrm{T}_{2}$ maps were generated from a series of spin echo images (repetition time $(\mathrm{TR})=3.0 \mathrm{~s}$ ) in which the echo time (TE) was logarithmically distributed in 10 steps from 9.7 to $100 \mathrm{~ms}$. The total experimental time was ca. $1 \mathrm{~h} . \mathrm{T}_{1}$ maps were generated from a series of inversion recovery spin echo images $(\mathrm{TR}=10.0 \mathrm{~s} ; \mathrm{TE}=9.7 \mathrm{~ms})$ in which the inversion recovery time was logarithmically distributed in 10 steps from $10 \mathrm{~ms}$ to $6.0 \mathrm{~s}$. The total experimental time was ca. $3.5 \mathrm{~h}$. The relaxation maps were calculated using a standard mono-exponential fit employing a least-squares procedure.

In vitro degradation of mMPIO by cathepsin L. Cathepsin L $(0.88 \mu \mathrm{g})$ in $98 \mu \mathrm{l}$ of citrate buffer $0.1 \mathrm{M} \mathrm{pH} 5.0$ containing $1 \mathrm{mM}$ EDTA was activated by addition of DTT $(2 \mu \mathrm{l}, 100 \mathrm{mM}$ in water). After $15 \mathrm{~min}$ at r.t., this pre-activated solution was added to $0.4 \mathrm{mg}$ of mMPIO in $400 \mu \mathrm{l}$ citrate buffer $\mathrm{pH}$ 5.0. The solution was incubated at $37^{\circ} \mathrm{C}$ and at selected time points $100 \mu \mathrm{l}$ of solution was taken and $1 \mu \mathrm{l}$ of the potent cysteine protease inhibitor E-64 (Sigma-Aldrich, UK) solution $1 \mathrm{mM}$ in DMSO was added. After $1 \mathrm{~h}$ at $4{ }^{\circ} \mathrm{C}$ the sample was diluted to $700 \mu \mathrm{l}$ and particle size measured.

In vitro macrophage uptake and degradation experiments. The synthesis of fluorescent-labelled mMPIO (with both L- and D-amino acid peptide linkers) and monomeric MPIO was performed as described in Supplementary Methods.
RAW264.7 murine macrophage cells (ATCC) were grown in DMEM supplemented with $10 \%$ FBS until ca. 50\% confluent. Cells were incubated with $1 \mu \mathrm{g}$ of multimeric particles (per $35 \mathrm{~mm}$ culture dish) for $30 \mathrm{~min}$ at $37^{\circ} \mathrm{C}$. Culture medium was replaced with fresh, pre-warmed complete Dulbecco's Modified Eagle's medium, and live cell experiments performed at $37^{\circ} \mathrm{C}$ using a Zeiss LSM510 laser scanning confocal equipped with a microscope incubator $\left(\mathrm{CO}_{2}=5 \%\right)$. Images were collected using a $63 \mathrm{X} 1.4 \mathrm{NA}$ objective, with the $488-\mathrm{nm}$ laser line of an argon laser used for fluorescence and transmitted light imaging. Twelve hours time courses were run; imaging interval, 2 min. Further experiments were performed in freshly isolated murine macrophages (Supplementary Methods) and the subcellular degradation process was monitored by TEM (Supplementary Methods).

In vivo mMPIO uptake and clearance experiments. Adult male NMRI mice $(30-40 \mathrm{~g})$ were anaesthetized and injected intravenously with $4 \mathrm{mg}$ Fe per kg body weight of (i) mMPIO- $\mathrm{NH}_{2}$ or (ii) equivalent sized amine-terminated monomeric MPIO. Animals were transcardially perfused with saline followed by $4 \%$ paraformaldehyde at either $1 \mathrm{~h}$ or 7 days ( $n=3$ per group) after microparticle injection. Two additional animals were injected with the same dose (4 mg Fe per $\mathrm{kg}$ ) of mMPIO-COOH and perfused 7 days post-injection. A further group of animals injected with the monomeric MPIO were perfused 14 days post-injection $(n=3)$. All in vivo experiments were approved by the UK Home Office.

Tissue samples from brain, heart, lung, kidney, liver, spleen and intestine were post-fixed for 7 days and paraffin wax embedded. Ten micrometre sections were dried overnight, de-waxed and stained for iron using Perls' Prussian Blue stain with a Nuclear Fast Red counterstain. For each tissue, three fields of view per animal at $\times 400$ magnification were analysed using a semiautomated thresholding method (ImageJ) to determine the percentage area of iron staining. Thresholding criteria, based on hue, saturation and luminosity values specific to the microparticles were constant for each tissue. Differences between groups were assessed using ANOVA followed by post-hoc pairwise $t$-tests with a Welch's correction for unequal variances where appropriate; group sizes were based on previous clearance experiments with commercial MPIO. Mice were assigned randomly to each group, and all analysis was performed blind to the sample groups.

Subsequently, a single dose extended acute toxicology study was commissioned from the Commercial Research Organization Sequani Ltd (full details in Supplementary Methods). The following assessments were made: body weight, organ weights, macroscopic pathology, haematology and blood chemistry. In addition, microscopic analysis, including assessment of iron deposition, was conducted on brain (at three levels), heart, kidney, liver, lung and spleen tissue. Subsequent in-house quantitative analysis of Perls' staining was performed as described above. Group sizes were determined by Sequani Ltd based on previous toxicology work, mice were assigned randomly to each group and analysis was performed blind to sample group.

Synthesis of fluorescently labelled, targeted mMPIOs. A solution of AlexaFluor 488 SDP ester $(2 \mathrm{mg}$ per $\mathrm{ml}, 250 \mu \mathrm{l})$ in DMSO was added to a solution of mMPIO-COOH $(5 \mathrm{ml}, 1 \mathrm{mg}$ Fe per $\mathrm{ml}$ ) in sodium bicarbonate buffer $0.1 \mathrm{M}$ $\mathrm{pH}$ 8.3. The reaction was shaken for $24 \mathrm{~h}$. Particles were collected using a Dynal magnet (Invitrogen, UK) and washed. Particles were resuspended in $900 \mu \mathrm{l}$ of MES buffer $0.1 \mathrm{M} \mathrm{pH}$ 6.0. SulfoNHS $(2 \mu \mathrm{l}, 1 \mathrm{M})$ in water and $\operatorname{EDC}(3.5 \mu \mathrm{l}, 0.1 \mathrm{M})$ in water were sequentially added to $180 \mu \mathrm{l}$ of the AF488-mMPIO solution. The resultant solution was stirred for $15 \mathrm{~min}$ at room temperature and then $400 \mu \mathrm{l}$ of rat anti-mouse VCAM-1 (clone $\mathrm{M} / \mathrm{K} 2$ ) or rat $\mathrm{IgG}_{2 \mathrm{a}}$ isotype control antibody (clone $\mathrm{KLH} / \mathrm{G} 2 \mathrm{a}-1-1)\left(0.5 \mathrm{mg} \mathrm{ml}^{-1}\right)$ followed by $400 \mu \mathrm{l}$ of sodium bicarbonate buffer $0.1 \mathrm{M} \mathrm{pH} 8.3$ was added. The sample was shaken for $24 \mathrm{~h}$. Particles were collected using a magnet (Invitrogen) washed and resuspended in $200 \mu \mathrm{l}$ of PBS buffer. The antibody loading was determined on multimeric particles from fluorescence intensity using Qifikit calibration beads (Dako, UK) according to the manufacturer's protocol, but substituting the provided fluorescently conjugated antibody with AlexaFluor594-conjugated rabbit anti-rat IgG $(\mathrm{H}+\mathrm{L})$ (Invitrogen; Cat. Nr. A-21211). mMPIO containing $5 \mu \mathrm{g}$ iron were diluted in $200 \mu \mathrm{l}$ of $1 \times \mathrm{PBS} / 0.1 \%$ Tween-20. AlexaFluor594-conjugated goat anti-mouse IgG $(\mathrm{H}+\mathrm{L})$ (Invitrogen; Cat. Nr. A-11005) $(8 \mu \mathrm{g})$ was added and incubated at $4{ }^{\circ} \mathrm{C}$ for $1 \mathrm{~h}$. The particles were pelleted on a Dynal magnetic separator (Invitrogen) and washed three times with $1 \times$ PBS/ $/ 1 \%$ Tween 20 . Flow cytometry experiments were performed on a BD LSRII flow cytometer and the data analysed using Flow Jo (Tree Star Inc., OR, USA).

In vitro antibody-mMPIO binding experiments. Murine endothelial (sEnd.1, PMID: 2736622) cells, cultured in $35 \mathrm{~mm}$ dishes cultured in $35 \mathrm{~mm}$ dishes (Corning, USA), were stimulated with recombinant mouse tumor necrosis factor- $\alpha$ for $8 \mathrm{~h}$, fixed with $4 \%$ formaldehyde for $10 \mathrm{~min}$ at room temperature, washed with PBS and stored at $4{ }^{\circ} \mathrm{C} . \alpha$ VCAM-AF488-mMPIO (1.625 $\mu \mathrm{g}$ Fe per ml), IgG-AF488mMPIO $(1.625 \mu \mathrm{g} \mathrm{Fe} \mathrm{per} \mathrm{ml)} \mathrm{or} \mathrm{PBS} \mathrm{was} \mathrm{added} \mathrm{to} \mathrm{cells} \mathrm{and} \mathrm{these} \mathrm{were} \mathrm{placed}$ on a bench-top rocker for $5 \mathrm{~min}$ at r.t. prior to thorough washing with PBS. Particle binding events were visualized using a $\times 40,0.6 \mathrm{NA}$ objective fitted to an Olympus IX-71 microscope. 
In vivo anti-VCAM-1-targeted experiments. Adult male NMRI mice (30-40 g or $10-12$ weeks, $n=3$ ) were anaesthetized with $2.0-2.5 \%$ isoflurane in $70 \% \mathrm{~N}_{2} \mathrm{O}: 30 \% \mathrm{O}_{2}$ and stereotaxically microinjected in the left striatum (co-ordinates from Bregma: anterior $0.5 \mathrm{~mm}$, lateral $2 \mathrm{~mm}$, depth $2.5 \mathrm{~mm}$ ) with $20 \mathrm{ng}$ of recombinant mouse IL- $1 \beta$ in $1 \mu \mathrm{l}$ low endotoxin saline $(n=3)$. After $3.1 \pm 0.1 \mathrm{~h}$, mice were re-anaesthetized and injected via a tail vein with $\alpha$ VCAM-AF488-mMPIO (4 mg Fe per $\mathrm{kg}$ ). Three control mice were studied: (i) naïve mouse injected intravenously with $\alpha$ VCAM-AF488-mMPIO as above; (ii) mouse injected intracerebrally with vehicle ( $1 \mu \mathrm{l}$ saline) and intravenously with $\alpha$ VCAM-AF488-mMPIO after $3.2 \mathrm{~h}$; and (iii) mouse injected intracerebrally with $20 \mathrm{ng}$ IL-1 $\beta$ in $1 \mu \mathrm{l}$ saline and intravenously with the non-targeted IgG-AF488-mMPIO after $3.0 \mathrm{~h}$. Following mMPIO injection, animals were positioned in a quadrature birdcage coil. During MRI, anaesthesia was maintained with $1.5-1.8 \%$ isoflurane, and electrocardiogram was monitored and body temperature maintained at $\sim 37^{\circ} \mathrm{C}$. All in vivo experiments were approved by the UK Home Office.

Magnetic resonance imaging. $\mathrm{A}_{2}{ }^{*}$-weighted three-dimensional gradient echo data set was acquired: flip angle $=27^{\circ}$; $\mathrm{TR}=65 \mathrm{~ms}$; TE $=7.5 \mathrm{~ms}$; field of view $($ FOV $)=11.2 \mathrm{~mm} \times 22.5 \mathrm{~mm} \times 22.5 \mathrm{~mm}$; matrix size, $96 \times 192 \times 256$; number of averages $=2$; total acquisition time $\sim 40 \mathrm{~min}$. Mid-point of acquisition was $1.6 \pm 0.4 \mathrm{~h}$ after microparticle injection. Data were zero-filled to $128 \times 256 \times 256$; final isotropic resolution $\sim 88 \mu \mathrm{m}$. Brains were masked, thresholded and MPIO binding quantified. Spin-echo $\mathrm{T}_{1}$-weighted images $(\mathrm{TR}=500 \mathrm{~ms} ; \mathrm{TE}=20 \mathrm{~ms}$; FOV $25 \mathrm{~mm} \times 25 \mathrm{~mm}$; matrix size, $128 \times 128$ ) were acquired pre- and $5 \mathrm{~min}$ post-Gd-DTPA injection (Omniscan; GE Healthcare, UK; $30 \mu$ l, i.v.). Each $\mathrm{T}_{2}{ }^{*}$-weighted data set was converted into tiff images, manually masked to exclude extracerebral structures and converted to 8-bit greyscale in Adobe Photoshop (Adobe Systems Incorporated, UK). The images were thresholded at a consistent level in the grey channel, such that any pixels of signal intensity $>3$ s.d. below the mean intensity of normal brain were set to zero (black) and all others were set to 1 (white), see Supplementary Fig. 39. The absolute level of thresholding varied between data sets according to variations in signal-to-noise. Signals arising from ventricles or sinuses were excluded by comparison to a naive animal imaged with no contrast agent, in which these structures appear hypointense naturally.

The masked and thresholded images were subsequently imported into ImagePro (Media Cybernetic, UK) and stacked into a single sequence. mMPIO binding, defined as all pixels with signal levels of zero, was quantified in 160 consecutive brain slices for each animal. Analysis was performed blind to the origin of the dataset. Segmented images were reconstructed using the three-dimensional Constructor plug-in to visualize the spatial distribution of binding, with low-signal areas assigned to the red channel and the anatomical image to the green channel. Voxel volumes were summed and expressed as raw volumes in microlitres with no surface rendering or smoothing effects. Since these were proof-of-principle experiments, to demonstrate the in vivo contrast effects of the mMPIO, only a sufficient number of animals were studied to demonstrate that these effects were reproducible and statistical analysis was not performed.

Immunohistochemistry and immunofluorescence. Following MRI, animals were transcardially perfused and the brains post-fixed, cryoprotected, embedded and frozen in isopentane. Expression of VCAM-1 and co-localization with mMPIO was verified both by immunofluorescence microscopy and immunohistochemically (rat anti-mouse VCAM-1 (clone M/K2)) using avidin-biotin complex amplification $^{22}$. To verify co-localization of the MMPIO with VCAM-1 expression, Prussian-blue staining to detect iron oxide was performed. Following completion of VCAM-1 immunohistochemistry, some slides were incubated with $20 \%$ hydrochloric acid and $10 \%$ potassium hexacyanoferrate(II) trihydrate for $20 \mathrm{~min}$ at $37^{\circ} \mathrm{C}$. After washing with PBS, the slices were then counterstained with cresyl violet.

Microscopy. Images were acquired using either an inverted epifluorescence microscope (DM IRB; Leica Microsystems, Wetzlar, Germany) or an inverted confocal microscope (LSM-710; Carl Zeiss Microimaging, Jena, Germany) and analysed using Image J and Zen (Carl Zeiss) software.

The LSM-710 confocal microscope was equipped with PC-Apochromat $\times 631.2 \mathrm{NA}$ oil immersion objective lens. Detection ranges were set to eliminate crosstalk between fluorophores: $409-485 \mathrm{~nm}$ for DAPI, $494-553 \mathrm{~nm}$ for AlexaFluor 488 and 564-712 nm for Cy3. The set of dichroics MBS 488/561 and MBS-405 were used. The 32 PMT array of the confocal was used to record lambda images that were subsequently unmixed with the Zen software using individually recorded spectra, thus removing inherent autofluorescent signal from the tissue.

Data availability. The particle sizing, MRI, microscope images and HPLC data that support the findings of this study are available in Oxford University ORA data system with the identifier https://doi.org/10.5287/bodleian:qaY9QE2kN. Other data that support the findings of this study are available from the corresponding authors upon request.

\section{References}

1. Gupta, A. K. \& Gupta, M. Synthesis and surface engineering of iron oxide nanoparticles for biomedical applications. Biomaterials 26, 3995-4021 (2005).

2. Barry, S. E. Challenges in the development of magnetic particles for therapeutic applications. Int. J. Hyperthermia 24, 451-466 (2008).

3. Laurent, S. et al. Magnetic iron oxide nanoparticles: synthesis, stabilization, vectorization, physicochemical characterizations, and biological applications. Chem. Rev. 108, 2064-2110 (2008).

4. Weissleder, R. et al. Ultrasmall superparamagnetic iron oxide: an intravenous contrast agent for assessing lymph nodes with MR imaging. Radiology 175, 494-498 (1990).

5. Weissleder, R., Bogdanov, A., Neuwelt, E. A. \& Papisov, M. Long-circulating iron oxides for MR imaging. Adv. Drug Deliv. Rev. 16, 321-334 (1995).

6. Kievit, F. M. \& Zhang, M. Surface engineering of iron oxide nanoparticles for targeted cancer therapy. Acc. Chem. Res. 44, 853-862 (2011).

7. Lau, J. F., Anderson, S. A., Adler, E. \& Frank, J. A. Imaging approaches for the study of cell-based cardiac therapies. Nat. Rev. Cardiol. 7, 97-105 (2010).

8. Haun, J. B., Yoon, T.-J., Lee, H. \& Weissleder, R. Magnetic nanoparticle biosensors. Wiley Interdiscip. Rev. Nanomed. Nanobiotechnol. 2, 291-304 (2010).

9. Perez, J. M., Josephson, L., O’Loughlin, T., Hogemann, D. \& Weissleder, R. Magnetic relaxation switches capable of sensing molecular interactions. Nat. Biotechnol. 20, 816-820 (2002).

10. Cheon, J. \& Lee, J.-H. Synergistically integrated nanoparticles as multimodal probes for nanobiotechnology. Acc. Chem. Res. 41, 1630-1640 (2008).

11. McLachlan, S. J. et al. Phase I clinical evaluation of a new iron oxide MR contrast agent. J. Magn. Reson. Imaging 4, 301-307 (1994).

12. Yang, Y., Yang, Y., Yanasak, N., Schumacher, A. \& Hu, T. C. C. Temporal and noninvasive monitoring of inflammatory-cell infiltration to myocardial infarction sites using micrometer-sized iron oxide particles. Magn. Reson. Med. 63, 33-40 (2010).

13. Weissleder, R., Kelly, K., Sun, E. Y., Shtatland, T. \& Josephson, L. Cell-specific targeting of nanoparticles by multivalent attachment of small molecules. Nat. Biotechnol. 23, 1418-1423 (2005).

14. Tassa, C. et al. Binding affinity and kinetic analysis of targeted small moleculemodified nanoparticles. Bioconjugate Chem. 21, 14-19 (2009).

15. Akhtar, A. M. et al. In vivo quantification of VCAM-1 expression in renal ischemia reperfusion injury using non-invasive magnetic resonance molecular imaging. PLoS ONE 5, e12800 (2010).

16. McAteer, M. A. et al. In vivo magnetic resonance imaging of acute brain inflammation using microparticles of iron oxide. Nat. Med. 13, 1253-1258 (2007).

17. van Kasteren, S. I. et al. Glyconanoparticles allow pre-symptomatic in vivo imaging of brain disease. Proc. Natl Acad. Sci. USA 106, 18-23 (2009).

18. von zur Muhlen, C. et al. Magnetic resonance imaging contrast agent targeted toward activated platelets allows in vivo detection of thrombosis and monitoring of thrombolysis. Circulation 118, 258-267 (2008).

19. McAteer, M. A. et al. Magnetic resonance imaging of endothelial adhesion molecules in mouse atherosclerosis using dual-targeted microparticles of iron oxide. Arterioscler. Thromb. Vasc. Biol. 28, 77-83 (2008).

20. Hoyte, L. C. et al. Molecular magnetic resonance imaging of acute vascular cell adhesion molecule-1 expression in a mouse model of cerebral ischemia. J. Cereb. Blood Flow Metab. 30, 1178-1187 (2010).

21. von zur Muhlen, C. et al. A contrast agent recognizing activated platelets reveals murine cerebral malaria pathology undetectable by conventional MRI. J. Clin. Invest. 118, 1198-1207 (2008).

22. Serres, S. et al. Molecular MRI enables early and sensitive detection of brain metastases. Proc. Natl Acad. Sci. USA 109, 6674-6679 (2012).

23. Kanke, M., Simmons, G. H., Weiss, D. L., Bivins, B. A. \& Deluca, P. P. Clearance of ${ }^{141} \mathrm{Ce}$-labeled microspheres from blood and distribution in specific organs following intravenous and intraarterial administration in beagle dogs. J. Pharmaceut. Sci. 69, 755-762 (1980).

24. Illum, L. et al. Blood clearance and organ deposition of intravenously administered colloidal particles. The effects of particle size, nature and shape. Int. J. Pharm. 12, 135-146 (1982).

25. Normann, S. in The Reticuloendothelial System: A Comprehensive Treatise, Vol. 7A Physiology (eds Reichard, S. M. \& Filkins, J. P.) 73-101 (Springer, 1984).

26. Sun, E. Y., Josephson, L., Kelly, K. A. \& Weissleder, R. Development of nanoparticle libraries for biosensing. Bioconjugate Chem. 17, 109-113 (2006).

27. Doronina, S. O. et al. Development of potent monoclonal antibody auristatin conjugates for cancer therapy. Nat. Biotechnol. 21, 778-784 (2003).

28. Leriche, G., Chisholm, L. \& Wagner, A. Cleavable linkers in chemical biology. Bioorg. Med. Chem. 20, 571-582 (2012). 
29. Mohamed, M. M. \& Sloane, B. F. Cysteine cathepsins: multifunctional enzymes in cancer. Nat. Rev. Cancer 6, 764-775 (2006).

30. Weissleder, R. et al. Superparamagnetic iron oxide: pharmacokinetics and toxicity. Am. J. Roentgenol. 152, 167-173 (1989).

31. Chouly, C., Pouliquen, D., Lucet, I., Jeune, J. J. \& Jallet, P. Development of superparamagnetic nanoparticles for MRI: effect of particle size, charge and surface nature on biodistribution. J. Microencapsul. 13, 245-255 (1996).

32. Schulze, E. et al. Cellular uptake and trafficking of a prototypical magnetic iron oxide label in vitro. Invest. Radiol. 30, 604-610 (1995).

33. Rawlings, N. D., Barrett, A. J. \& Bateman, A. MEROPS: the peptidase database. Nucleic Acids Res. 38, D227-D233 (2010).

34. Cotrin, S. S. et al. Positional-scanning combinatorial libraries of fluorescence resonance energy transfer peptides to define substrate specificity of carboxydipeptidases: assays with human cathepsin B. Anal. Biochem. 335, 244-252 (2004).

35. Choe, Y. et al. Substrate profiling of cysteine proteases using a combinatorial peptide library identifies functionally unique specificities. J. Biol. Chem. 281, 12824-12832 (2006).

36. Staros, J. V. N-hydroxysulfosuccinimide active esters: bis(N-hydroxysulfosuccinimide) esters of two dicarboxylic acids are hydrophilic, membraneimpermeant, protein cross-linkers. Biochemistry 21, 3950-3955 (1982).

37. Terada, M. A novel role in the removal of blood-borne foreign bodies for pulmonary capillaries in the guinea pig. Virchows Arch. B Cell Pathol. Incl. Mol. Pathol. 63, 147-157 (1993).

38. Pittet, M. J., Swirski, F. K., Reynolds, F., Josephson, L. \& Weissleder, R. Labeling of immune cells for in vivo imaging using magnetofluorescent nanoparticles. Nat. Protocols 1, 73-79 (2006).

\section{Acknowledgements}

This work was funded by a Cancer Research UK Programme Grant-C5255/A12678 (to N.R.S.), MRC DPFS Grant G0902181 (to N.R.S., D.C.A., R.P.C. and B.G.D.),

a Research Grant from Glycoform Limited (to B.G.D.), an Isis Innovation Fund Award (to D.C.A., B.G.D. and S.I.v.K.), an EPSRC Platform Grant EP/E000614/1 (to B.G.D.), a Royal Society Wolfson Research Merit Award (to B.G.D.) and a Senior Fellowship from The Wellcome Trust (to R.P.C.). We thank Dr Jo Peach for helpful discussions in the early stages of concept development, Mick Woodcock for flow cytometry experiments, Dr Michael Stratford for his help with the HPLC-MS measurements and Dr Lisa Folkes for help with HPLC analyses.

\section{Author contributions}

D.C.A., R.P.C., B.G.D., S.I.v.K. and N.R.S. jointly conceived the need for a biodegradable, low-density MPIO with the clearance characteristics and size range of the particles described herein. D.C.A., B.G.D., S.I.v.K. devised a molecular strategy for synthesis of such a particle. B.G.D., S.I.v.K., A.A.A.A., K.W., G.B. and F.P.-B. were responsible for the particle construction, including physicochemical analysis, characterization, degradation kinetics and enzymatic processing. F.P.-B., S.S.L., G.B. and F.D.S. performed peptide specificity experiments. F.P.-B. and A.A.A.A. optimized the particle synthesis and characterization, and synthesized compounds for the biological studies. A.A.K., F.P.-B. and N.R.S. performed the relaxivity experiments. D.C.A., R.P.C., B.G.D. and N.R.S. designed the biological studies. A.J., F.P.-B. and K.W. performed the cellular microscopy experiments. S.J.C. and N.R.S. designed the formal toxicology study, which was conducted by Sequani Ltd. D.C.A., N.R.S. and M.S.S. performed the in vivo biodistribution experiments. D.C.A., J.R.L., M.S.S., S.S. and N.R.S. performed the in vivo MRI experiments. C.B., J.R.L. and M.S.S. conducted the histological measurements on resulting tissue samples. D.C.A., B.G.D., A.J., J.R.L., F.P.-B., S.S., M.S.S. and N.R.S. analysed the biological data. D.C.A., S.J.C., R.P.C., B.G.D., S.I.v.K., F.P.-B. and N.R.S prepared the manuscript. All authors read and edited the manuscript.

\section{Additional information}

Supplementary Information accompanies this paper at http://www.nature.com/ naturecommunications

Competing financial interests: D.C.A., B.G.D. and N.R.S. declare that a patent (WO2008035069) associated with this work has been filed. The remaining authors declare no competing financial interests.

Reprints and permission information is available online at http://npg.nature.com/ reprintsandpermissions/

How to cite this article: Perez-Balderas, F. et al. Covalent assembly of nanoparticles as a peptidase-degradable platform for molecular MRI. Nat. Commun. 8, 14254 doi: $10.1038 /$ ncomms14254 (2017)

Publisher's note: Springer Nature remains neutral with regard to jurisdictional claims in published maps and institutional affiliations.

This work is licensed under a Creative Commons Attribution 4.0 International License. The images or other third party material in this article are included in the article's Creative Commons license, unless indicated otherwise in the credit line; if the material is not included under the Creative Commons license, users will need to obtain permission from the license holder to reproduce the material. To view a copy of this license, visit http://creativecommons.org/licenses/by/4.0/

(C) The Author(s) 2017 